\title{
Targeted fascicular biopsy of the sciatic nerve and its major branches: rationale and operative technique
}

\author{
Stepan Capek, MD, ${ }^{1,2}$ Kimberly K. Amrami, MD, ${ }^{3}$ P. James B. Dyck, MD, ${ }^{4}$ and \\ Robert J. Spinner, MD'1 \\ Departments of ${ }^{1}$ Neurosurgery, ${ }^{3}$ Radiology, and ${ }^{4}$ Neurology, Mayo Clinic, Rochester, Minnesota; and ${ }^{2}$ International Clinical \\ Research Center, St. Anne's University Hospital Brno, Brno, Czech Republic
}

\begin{abstract}
OBJECT Nerve biopsy is typically performed in distal, noncritical sensory nerves without using imaging to target the more involved regions. The yield of these procedures rarely achieves more than $50 \%$. In selected cases where preoperative evaluation points toward a more localized (usually a more proximal) process, targeted biopsy would likely capture the disease. Synthesis of data obtained from clinical examination, electrophysiological testing, and MRI allows biopsy of a portion of the major mixed nerves safely and efficiently. Herein, experiences with the sciatic nerve are reported and a description of the operative technique is provided.
\end{abstract}

METHODS All cases of sciatic nerve biopsy performed between 2000 and 2014 were reviewed. Only cases of fascicular nerve biopsy approached from the buttock or the posterior aspect of the thigh were included. Demographic data, clinical presentation, and the presence of percussion tenderness for each patient were recorded. Reviewed studies included electrodiagnostic tests and imaging. Previous nerve and muscle biopsies were noted. All details of the procedure, final pathology, and its treatment implications were recorded. The complication rate was carefully assessed for temporary as well as permanent complications.

RESULTS One hundred twelve cases (63 men and 49 women) of sciatic nerve biopsy were performed. Mean patient age was 46.4 years. Seventy-seven (68.8\%) patients presented with single lower-extremity symptoms, $16(14.3 \%)$ with bilateral lower-extremity symptoms, and $19(17 \%)$ with generalized symptoms. No patient had normal findings on physical examination. All patients underwent electrodiagnostic studies, the findings of which were abnormal in $110(98.2 \%)$ patients. MRI was available for all patients and was read as pathological in 111 (99.1\%). The overall diagnostic yield of biopsy was $84.8 \%(n=95)$. The pathological diagnoses included inflammatory demyelination, perineurioma, nonspecific inflammatory changes, neurolymphomatosis, amyloidosis, prostate cancer, injury neuroma, neuromuscular choristoma, sarcoidosis, vasculitis, hemangiomatosis, arteriovenous malformation, fibrolipomatous hamartoma (lipomatosis of nerve), and cervical adenocarcinoma. The series included 11 (9.9\%) temporary and 5 (4.5\%) permanent complications: 3 patients $(2.7 \%)$ reported permanent numbness in the peroneal division distribution, and 2 patients $(1.8 \%)$ were diagnosed with neuromuscular choristoma that developed desmoid tumor at the biopsy site 3 and 8 years later.

CONCLUSIONS Targeted fascicular biopsy of the sciatic nerve is a safe and efficient diagnostic procedure, and in highly selected cases can be offered as the initial procedure over distal cutaneous nerve biopsy. Diagnoses were very diverse and included entities considered very rare. Even for the more prevalent diagnoses, the biopsy technique allowed a more targeted approach with a higher diagnostic yield and justification for more aggressive treatment. In this series, new radiological patterns of some entities were identified, which could be biopsied less frequently.

http://thejns.org/doi/abs/10.3171/2015.6.FOCUS15213

KEY WORDS targeted biopsy; fascicular biopsy; sciatic nerve; surgery; technique

$\mathrm{N}$ ERVE biopsy is an established part of the evaluation of peripheral nerve disease. Most peripheral neuropathies are length-dependent disorders with the most distal nerves being more severely involved. Therefore, it is appropriate that the most commonly biopsied nerves are distal cutaneous nerves such as the sural nerve.
Depending on the clinical presentation, other nerves, including the superficial peroneal nerve in the lower limb and the superficial radial nerve in the upper limb, may be biopsied. In general, biopsied nerves are accessible and noncritical sensory nerves; sacrifice leaves a tolerable deficit, including pain and sensory loss in the distribution

ABBREVIATIONS CIDP = chronic inflammatory demyelinating polyneuropathy; SPGR = spoiled gradient echo recalled

SUBMITTED April 29, 2015. ACCEPTED June 9, 2015.

INCLUDE WHEN CITING DOI: 10.3171/2015.6.FOCUS15213. 
of the nerve. In patients presenting with predominantly motor symptoms, some groups routinely biopsy mixed or pure motor nerves such as the entire superficial peroneal nerve, ${ }^{2}$ the obturator nerve branch to the gracilis muscle, ${ }^{1}$ or the pronator branch of the median nerve. ${ }^{6}$ The diagnostic yield is difficult to assess due to diverse methodology and different spectrum of conditions selected for biopsy, but ranges from $35 \%$ to $50 \%{ }^{8,19,21}$ for sural nerve biopsies. One explanation for the relatively low yield is easy: not all diseases affect all nerves. A more targeted biopsy would likely capture a more localized process and improve the diagnostic yields for selected cases with more focal and proximal involvement.

During the past 2 decades, imaging of the peripheral nerves has vastly improved; abnormalities of the nerve may be observed, though not understood. Oberlin et al. ${ }^{18}$ demonstrated that individual fascicles could be taken even from major mixed nerves without causing significant deficit. On these premises, we adapted a fascicular biopsy technique of the sural nerve ${ }^{7}$ and combined it with the principles employed in image-guided biopsy used in the central nervous system. ${ }^{20}$ We have developed a specialized clinical practice with emphasis on clinical, electrophysiological, imaging, and pathological features. We believe that synthesizing all data obtained from clinical examination, electrophysiological testing, and high-resolution MRI allow us to select better, higher yield targets and biopsy them safely. We have previously described this multidisciplinary approach from a clinical, radiographic, and surgical vantage, but here we emphasize the surgical technique., ${ }^{4,22}$ We provide a summary of our experience over a 15-year period with the most common site of biopsy, the sciatic nerve, and our improved results in obtaining diagnoses for otherwise undiagnosed cases.

\section{Methods \\ Evaluation}

The selection of the biopsy site is based on careful analysis of the preoperative data from the clinical history, physical examination, electrodiagnostic testing, and imaging findings. The clinical history and physical examination gives an initial impression on localization and extent of the disease (e.g., atrophy of the gluteal musculature or involvement of the posterior femoral cutaneous nerve points toward a lumbosacral plexopathy and, if the sciatic nerve is involved, then probably the most proximal sampling at the sciatic notch will have the highest yield). When present, the point of maximal percussion tenderness (Tinel's sign) is used as an important sign and good correlator of the location of pathology. Nerve conduction studies and electromyography further specifies the character of the sensory and motor deficits; absent sensory nerve conduction potentials means the deficits are distal to the dorsal root ganglion. Nerve conduction studies can also provide information on the presence and degree of the conduction block. Other testing, such as quantitative sensory testing, autonomic reflex testing, and thermoregulatory sweat testing, is also helpful for identifying the populations of neurons involved and the level of their involvement. Preoperative evaluation also includes high- resolution 3-T MRI. T2-weighted (e.g., fast spin echo) and T1-weighted or gradient echo-based (e.g., spoiled gradient echo recalled [SPGR]) sequences with very robust fat suppression, with and without gadolinium enhancement, have become part of our standardized peripheral nerve imaging protocol. These images should be interpreted specifically to describe not only what division but what groups of fascicles seem to be involved (e.g., the most lateral of the tibial division) and should provide a bony landmark where the maximum pathology is found (e.g., at the level of the ischial tuberosity) (Fig. 1). When a malignancy (such as neurolymphomatosis) is suspected, PET/CT can point out the "hot" areas of the nerves, although they are typically below the definite standard uptake values for malignancy.

\section{Operative Technique}

The site with the highest probability of pathology is selected preoperatively based on a combination of clinical, electrodiagnostic, and imaging findings. The patient is placed prone. General anesthesia is used, and long-acting nondepolarizing neuromuscular blockers are discouraged. In our experience, a 6- to 8-cm skin incision is sufficient in terms of safety and exposure. Depending on the level of the incision, a gluteal muscle or hamstring interval splitting approach is performed in the buttock or thigh, respectively. For sciatic notch exposure, the piriformis muscle can be preserved. Spine retractors facilitate exposure. The upper buttock area should be approached with great caution, especially in cases with extensive fibrosis and scarring (e.g., after radiation), and gluteal nerves and vessels should be preserved. The sciatic nerve is mobilized under magnification (i.e., external neurolysis) in a vasoloop. Additional exposure of the nerve can be obtained well beyond the skin incision if the skin and muscles are retracted. Internal neurolysis allows identification of the peroneal (small and lateral) and tibial divisions. Use of a disposable nerve stimulator (Vari-stim III Nerve locator, Medtronic Xomed) confirms the identity of the divisions. It also helps to identify nonfunctioning versus functioning fascicles. Since oftentimes the individual fascicles may not show any striking abnormalities apart from edema, one might rely on the location of the fascicular abnormality on MRI. Two fascicular groups (preferably, pathologically appearing ones) should be selected, typically from different areas of the nerve that are nonfunctioning when tested with a stimulator. Two fascicles provide sufficient, representative pathological material without undue risk of a postoperative deficit. Although interdigitations and connections may limit the length, the fascicles can typically be mobilized over $6-8 \mathrm{~cm}$ safely in this location of the nerve (Fig. 2). Intraneural dissection should be performed under magnification using either loupes or a microscope. Gentle irrigation with saline can help visualization. Each dissected fascicle is marked with a new vasoloop. A cutaneous branch (e.g., the sural communicating nerve or the posterior femoral cutaneous nerve) or a muscular branch (e.g., the hamstring branch) may provide additional material if encountered. Before resection, each fascicle should be retested with the portable stimulator. After fascicle resection, a suture is placed in the proximal end and a lead weight is attached to the distal end to preserve the fascicle 
length. For a 6- to 8-cm specimen, a 2- to 5-g weight is adequate based on the diameter. ${ }^{7}$ The resected fascicles are sent in transport containers filled with fixatives to the peripheral nerve lab without delay (Fig. 3; Video 1).

VIDEO 1. A step-by-step animation of the sciatic nerve fascicular biopsy procedure. After careful exposure and dissection, the sciatic nerve is tested with a portable stimulator and 2 fascicles are selected and carefully dissected. The fascicles are then retested, and if no motor response is elicited, they are resected and promptly sent to the peripheral nerve laboratory for pathological examination. Used with permission of Mayo Foundation for Medical Education and Research. All rights reserved. Click here to view with Media Player. Click here to view with Quicktime.

A small segment can be sent for frozen section if malignancy is suspected. If vasculitis is identified in the differential diagnosis, a small piece of epineurium can provide epineurial vessels for evaluation.

After completion of the biopsy, the wound is irrigated generously and inspected for bleeding. Hemostasis is achieved. The incision is closed respective of the anatomical layers. The procedure takes about 45 minutes, and patients can be discharged the same day or stay in the hospital overnight. Patients are presented with the final pathology often within 1 week.

\section{Series}

After securing an institutional review board approval, we reviewed all cases of sciatic nerve biopsy performed between 2000 and 2014 at our institution. Only cases of fascicular nerve biopsy approached from the buttock or the posterior aspect of the thigh were included. Cases of transabdominal intrapelvic lumbosacral plexus biopsy or nerves distal to the popliteal fossa were excluded. Patients with compression only or nerve masses were excluded. Demographic data (age, sex), clinical presentation (motor and sensory deficits, pain, laterality, distribution of symptoms), and the presence of percussion tenderness (local or radiating pain) were recorded for each patient. The reviewed studies included electrodiagnostic tests and imaging modalities, including MRI and PET/CT. Previous nerve and muscle biopsies were noted. The evaluated details of the sciatic nerve biopsy included site, approach, biopsied division, number of fascicles taken, length, appearance, and function as tested with intraoperative stimulation. Final and perioperative (when available) pathologies were reviewed. Treatment implications and postoperative complications of the biopsy were recorded.

\section{Results}

\section{Series Characteristics}

One hundred twelve patients (63 men and 49 women) were included in the study. The mean age was 46.4 years (range 2-85 years): 16 patients were children, and 96 patients were adults. All patients were evaluated by a neurologist (often P.J.B.D.) and 1 neurosurgeon specializing in the peripheral nerve (R.J.S.). The imaging studies were reviewed by a radiologist with experience in peripheral nerve imaging (K.K.A.). Forty-two patients (37.5\%) presented with left lower-extremity symptoms, 35 (31.3\%) with right lower-extremity symptoms, 16 (14.3\%) with bi-
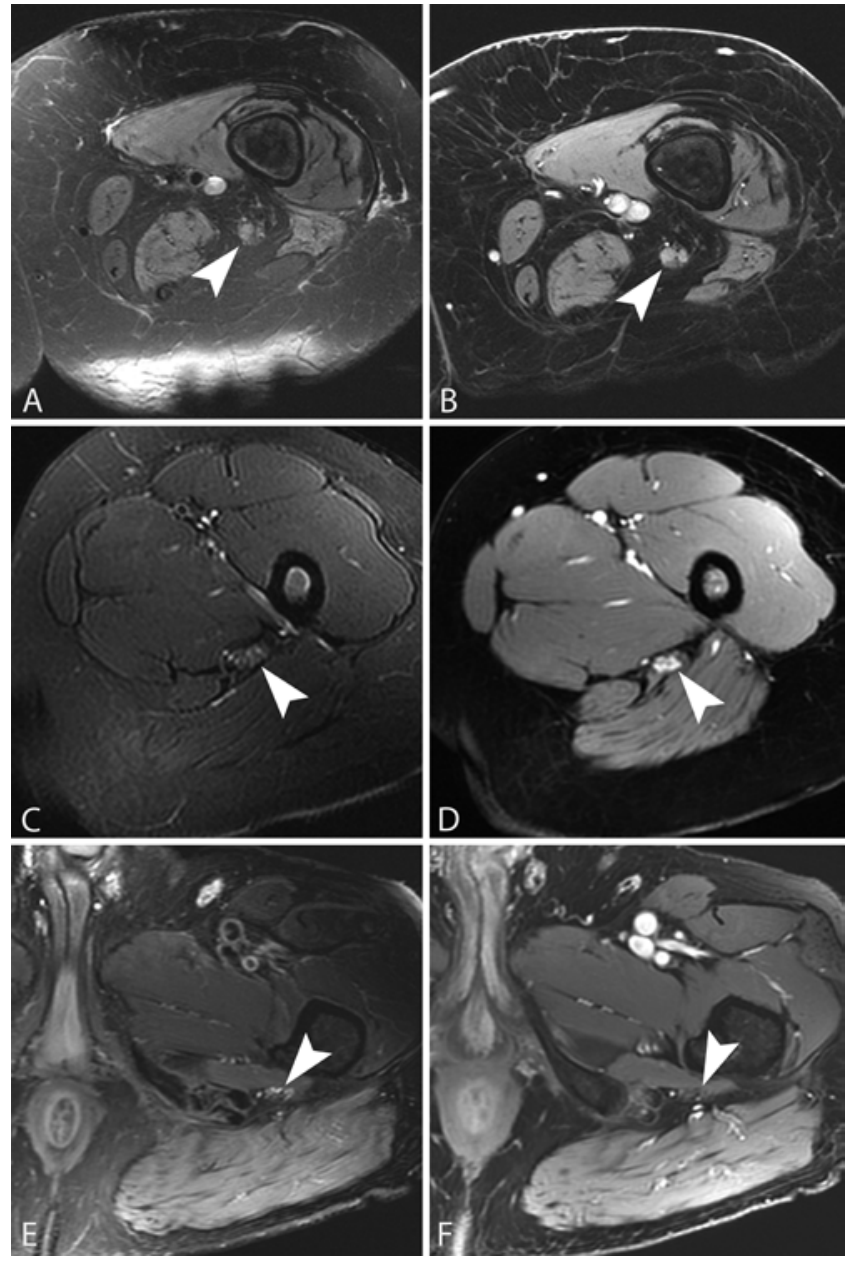

FIG. 1. Imaging findings. Several imaging patterns have been identified based on our experience with sciatic nerve biopsies. An axial T2weighted fat-suppressed image (A) of the left distal thigh in a patient with neurolymphomatosis demonstrates increased signal in the left sciatic nerve (arrowhead). An axial SPGR image (B) obtained at the same level demonstrating enhancement of the left sciatic nerve in the same patient (arrowhead). This pattern is frequently seen in patients with neurolymphomatosis, although it is nonspecific. In a patient with perineurioma, $(\mathbf{C})$ the sciatic nerve is typically isointense to slightly hyperintense to the surrounding musculature on T2-weighted images (arrowhead; fat-suppressed T2-weighted image), but (D) avidly enhancing on gadolinium-enhanced images (arrowhead; gadolinium-enhanced SPGR image). In contrast, (E) chronic inflammatory demyelinating polyneuropathy characteristically presents with hyperintense nerves on T2-weighted imaging (arrowhead; fat-suppressed T2-weighted image), but $(F)$ almost no enhancement is seen on gadolinium-enhanced images (arrowhead; gadolinium-enhanced SPGR image). In this instance, there is incomplete involvement of the sciatic nerve at the level of the ischial tuberosity.

lateral lower-extremity symptoms, and 19 (17\%) with generalized symptoms. Weakness was present in 110 patients (98.2\%), sensory loss in 94 (83.9\%; sensory loss could not be assessed in the 2 youngest patients), and radiating pain in $63(56.3 \%)$; 0 patients were asymptomatic. On physical examination, $34(30.4 \%)$ and $37(33 \%)$ patients demonstrated local and radiating pain (Tinel's sign) on percussion, respectively. The sciatic nerve was diffusely tender without the Tinel's sign in 1 patient $(0.9 \%)$. No patient had 

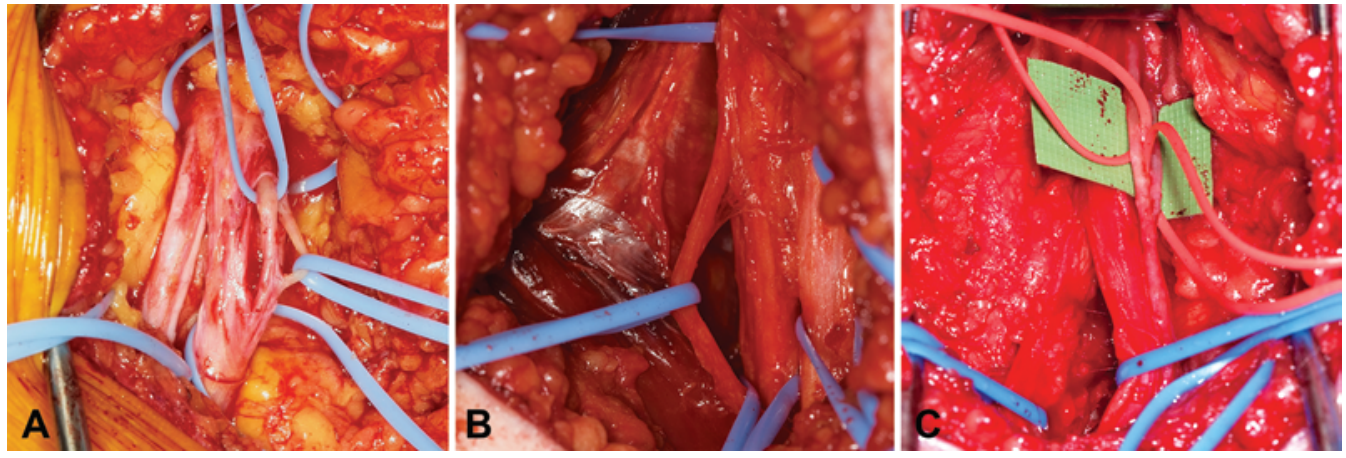

FIG. 2. Intraoperative photographs. Intraoperative photographs show irregularly appearing (A-C) and discolored (C) fascicles mobilized from the left sciatic nerves in patients with neurolymphomatosis $(A)$, perineurioma $(B)$, or chronic inflammatory demyelinating polyneuropathy $(C)$. These images were obtained from the same patients as the MR images shown in Fig. 1.

normal findings on physical examination. Electrodiagnostic studies revealed sciatic mononeuropathy in 37 patients (33\%), polyradiculoneuropathy in $30(26.8 \%)$, unilateral lumbosacral plexopathy in $29(25.9 \%)$, bilateral lumbosacral plexopathy in $12(10.7 \%)$, and multiple mononeuropathy in $1(0.9 \%)$. The electrodiagnostic examination was suggestive of anterior horn disease in $1(0.9 \%)$ patient and demonstrated normal findings in 2 patients (1.8\%). MRI was available for all patients and read as pathological in $111(99.1 \%)$ and normal in $1(0.9 \%)$ patient. The specific imaging findings associated with different diseases in the series are beyond the scope of this article. Twenty-two pa-

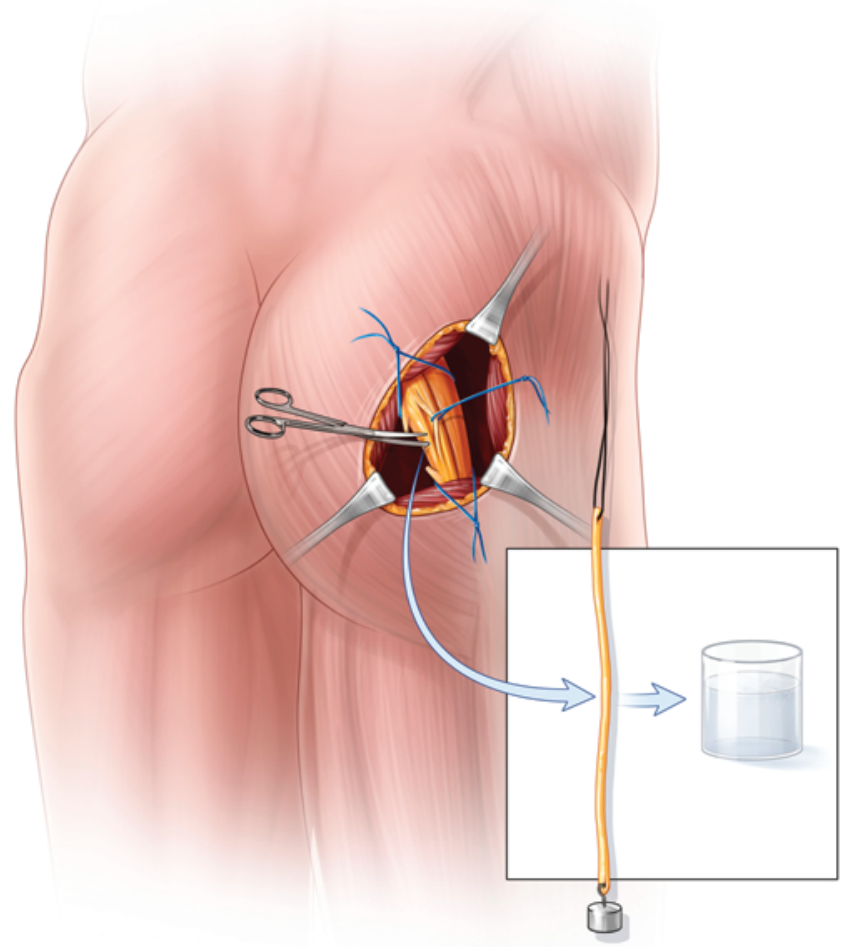

FIG. 3. Illustration. An artistic rendition of the last step of a targeted fascicular biopsy of the sciatic nerve. After careful selection and dissection, 2 fascicles are resected and promptly sent for pathological examination hanging by a suture at the proximal end and a lead weight attached to the distal end. Used with permission of Mayo Foundation for Medical Education and Research. All rights reserved. tients underwent PET/CT, which demonstrated increased uptake in the sciatic nerve in $5(22.7 \%)$ patients. Previous peripheral nerve biopsy was performed in $18(16.1 \%)$ patients, muscle biopsy in 3 (2.7\%), skin biopsy in $1(0.9 \%)$, and cauda equina biopsy in $1(0.9 \%)$. All these biopsies were nondiagnostic, except 5 sural nerve biopsies that showed nonspecific inflammation.

\section{Biopsies}

One hundred twelve biopsies of the sciatic nerve or its divisions were performed: left side in $58(51.8 \%)$ and right side in $50(44.6 \%)$ patients, the left tibial nerve in $3(2.7 \%)$, and the right tibial in $1(0.9 \%)$. The nerve was approached at the level of the sciatic notch (upper buttock approach) in $40(35.7 \%)$ patients and at the mid-buttock level in $12(10.7 \%)$. The lower buttock (at the level of the ischial tuberosity)-proximal thigh approach was used in $39(34.8 \%)$ patients, the midthigh approach in 7 (6.3\%), and the distal thigh or popliteal fossa approach in $14(12.5 \%)$. Only the tibial division was biopsied in 50 (44.6\%) patients, peroneal as well as tibial in 37 (33.0\%), only peroneal in $19(17 \%)$, and not specified in $6(5.4 \%)$. In addition to the biopsy of the sciatic nerve, 5 patients underwent biopsy of a hamstring branch, 2 of a posterior femoral cutaneous nerve, and 2 of a sural communicating nerve. In 3 patients, the nerve was released from extensive scarring, in 1 patient an arteriovenous malformation was cauterized, and in 1 patient a deltoid muscle biopsy was also performed. An intraoperative stimulator was used in 100 patients, but in 12 patients this information was not recorded. Of these, nonstimulating fascicles were resected in 94 patients, weakly functioning fascicles were selected in 2 patients, stimulation produced contraction of the nerve itself in 2 patients (both with neuromuscular choristomas), and, finally, the reaction to stimulation was not recorded in 2 patients. In $67(59.8 \%)$ patients, the fascicles looked grossly abnormal, and in $45(40.2 \%)$ patients no overt gross pathology was observed. On average, 2 fascicles were sampled (range 1-3) with an average length of $7.2 \mathrm{~cm}$ (range $4-14 \mathrm{~cm}$ ).

\section{Pathology and Treatment Implications}

Overall, the biopsy yielded a diagnosis in 95 patients (84.8\%). A separate paper emphasizing the different neuro- 
pathological diagnoses, treatments, and outcomes from targeted fascicular nerve biopsy is reported elsewhere, so these specifics are not presented here. However, some discussion about these diagnoses and the treatment implications is useful. The most common pathological finding from the sciatic biopsies was inflammatory demyelination. These biopsies often showed hypertrophic changes with large onion bulb formations (layers of Schwann cell processes from chronic demyelination and remyelination) and epineurial and endoneurial inflammatory cell collections (Fig. 4). Many of these patients were diagnosed with chronic inflammatory demyelinating polyradiculoneuropathy (CIDP) and started on new immunotherapy, or the prior treatment was escalated. The cases with perineurioma avoided unnecessary treatment, as this tumor does not respond to immunotherapy. Prospectively, eligible patients would be offered tendon transfer procedures. Patients whose biopsy showed only nonspecific inflammatory neuropathies were started on immunotherapy. Patients with microvasculitis (diabetic lumbosacral radiculoplexus neuropathy) were treated with steroids. Patients diagnosed with neurolymphomatosis were started on lymphoma treatment. One patient found to have amyloidosis was started on intravenous immunoglobulin for concomitant nerve inflammation. All patients with perineural spread of prostate or cervical cancer to the sciatic were referred for oncological treatment. Patients with neurosarcoidosis were treated with infliximab or steroids, and patients with vasculitis were started on steroids. No further treatment was pursued in cases of neuromuscular choristoma, injury neuroma, capillary hemangiomatosis, arteriovenous malformation, or fibrolipomatous hamartoma (lipomatosis of nerve).

\section{Outcome and Complications}

After the operation, pain improved in $4(3.6 \%)$ patients, but $8(7.1 \%)$ patients reported temporary radiating pain worse than their preoperative state. One patient developed a rash, 1 patient developed peroneal nerve weak-

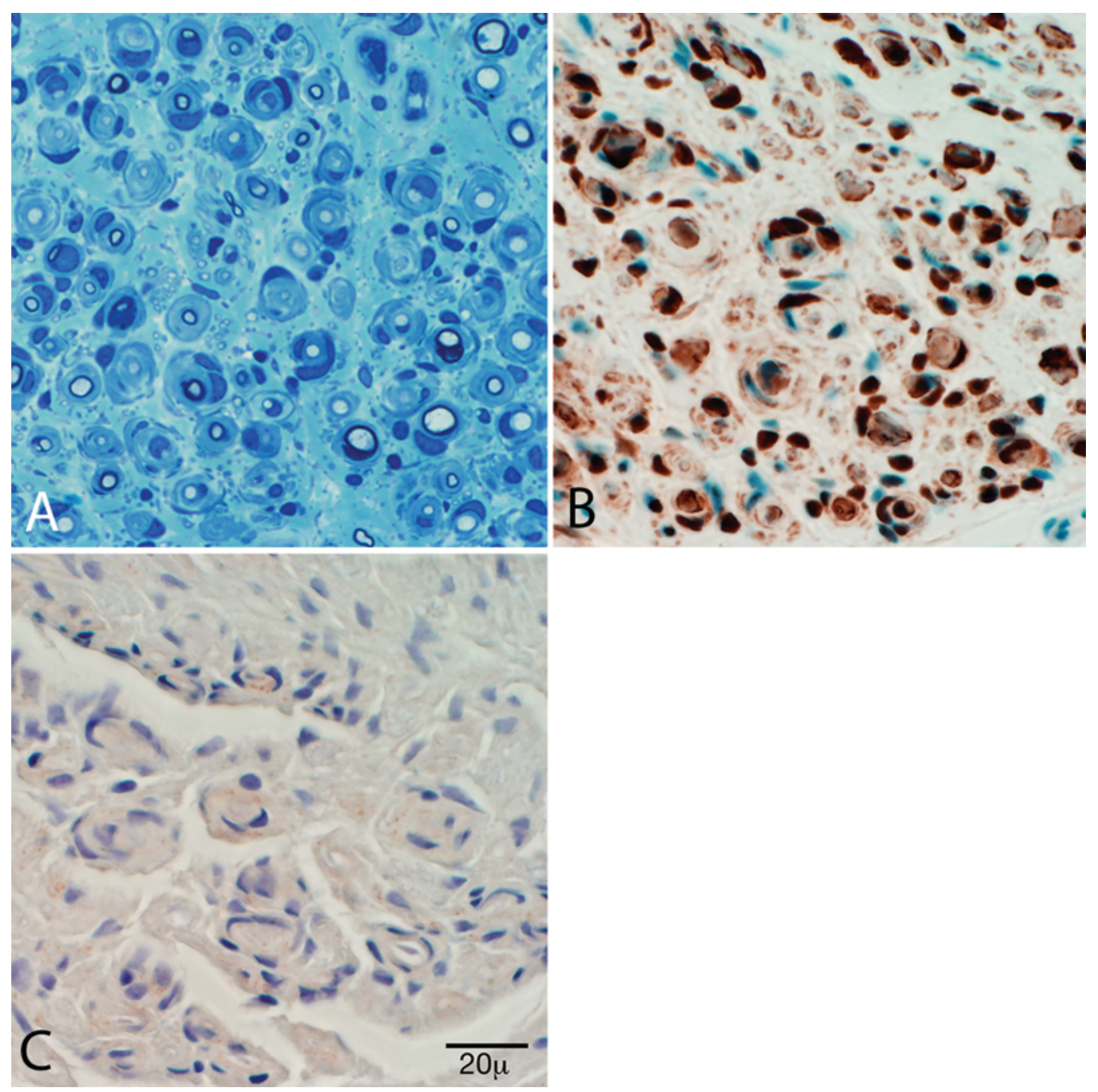

FIG. 4. Cross-sections from a fascicular sciatic nerve biopsy showing chronic demyelination. A: An epoxy section stained with methyl blue (original magnification $\times 650$ ) shows thinly myelinated nerve fibers surrounded by onion bulbs. B: A paraffin crosssection reacted to $S 100$ immunohistochemistry (original magnification $\times 650$ ) confirms that the onion bulbs are composed of Schwann cell processes. C: A paraffin section reacted to epithelial membrane antigen immunohistochemistry (original magnification $\times 650$ ), which shows no reactivity and excludes perineurial-derived pseudo-onion bulbs as are seen in perineurioma. 
ness, and 1 patient developed urinary retention, all of which resolved spontaneously within weeks after surgery. Paresthesias lasting several hours or days were reported fairly frequently, but were not considered bothersome by the patients and thus were not consistently reported in the documentation. Three patients $(2.7 \%)$ reported permanent numbness in the peroneal nerve distribution, which was not present preoperatively. Two patients (1.8\%) diagnosed with neuromuscular choristoma developed desmoid tumor at the biopsy site 3 and 8 years later. ${ }^{11,12}$

\section{Discussion}

Our experience of performing targeted fascicular biopsy on the sciatic nerve has been proven safe and efficient in providing valuable diagnostic information. The technique also highlights the need for collaboration among neurologists, radiologists, neurosurgeons, and pathologists. The variety of neurological disorders and subtle differences among them requires interest and expertise in the peripheral nerves across disciplines. Proof of this concept are the numerous MRI scans initially interpreted as normal, although on careful review by radiologist(s) with experience in peripheral nerve imaging, clear pathology was identified. These should not be interpreted as insufficient initial readings; it rather shows the complexity of peripheral nerve evaluation.

\section{Rationale and Indications}

Tissue Is the Issue

The main indication for targeted biopsy was disease refractory to treatment or following an atypical course, including focal neurological deficits. In these escalations, the treatment is frequently controversial without clear proof of the pathological process (e.g., high doses of immunotherapy [steroids, intravenous immunoglobulin, or others] for refractory CIDP come with significant side effects). In the second group are patients without a definite diagnosis despite previous extensive evaluation, sometimes including a nondiagnostic cutaneous nerve biopsy. The focal nature of the deficits is an initial clue that focal MRI imaging and subsequent biopsy of this region may be warranted. In these instances, the need for higher yield tissue is clear, especially when malignancy is concerned. On the other hand, inappropriate treatment of a misdiagnosed condition comes with an unnecessary risk of side effects; cost efficiency almost does not need mentioning. It is also important that the tissue once received goes to a laboratory with special interest in nerve pathology that is able to treat it appropriately and perform special preparations (such as teased nerve fibers). Many pathology labs do not have the necessary skill to handle the nerve well.

\section{The Benefit Should Outweigh the Risk}

Benefit. The diagnostic yield in our series was approximately $85 \%$, which is higher than in any other reported nerve biopsy series. This shows that with an appropriate selection of patients where the preoperative evaluation strongly points toward more localized, proximal pathology, it is beneficial to perform a targeted biopsy rather than a nontargeted distal biopsy. However, a careful risk- benefit assessment has to be performed should a sciatic nerve biopsy be offered as the initial procedure over a distal cutaneous nerve biopsy. With increased experience, we identified conditions with reproducible clinical and imaging patterns that we no longer initially biopsy (we consider biopsy for atypical or refractory cases). These include inflammatory demyelination (CIDP), ${ }^{3}$ perineurioma, ${ }^{3,16}$ lipomatosis of nerve,$^{15}$ and neuromuscular choristoma. ${ }^{17}$ Despite the high yield, the biopsy results were negative for cancer in 17 patients $(15.2 \%)$. We emphasize that negative biopsy results do not exclude the disease process. A high degree of suspicion should be maintained, especially in cancer patients. One patient in the series was a 65 -year-old old woman with a history of ovarian cancer. The patient's disease progressed and required systemic treatment despite the negative biopsy result. We still believe her nerve symptoms were due to tumor invasion.

Risk. The safety of targeted fascicular biopsy is based on the preoperative selection of the target (nerve), functional status of the nerve, number of fascicles biopsied, knowledge of (intra)neural anatomy, and use of an intraoperative stimulator. The aim is to perform the least risky procedure, both in terms of possible postoperative deficits and procedural risk itself. Always take as few fascicles as necessary, as additional fascicles may increase yield but will increase risk. The lesions of the lumbosacral plexus frequently involve the intradural nerve roots as well as the proximal sciatic nerve. We find the sciatic nerve at the sciatic notch to be very representative of lumbosacral pathology and much easier and safer to approach than to perform a cauda equina biopsy or transabdominal lumbosacral plexus biopsy. Furthermore, only nonfunctioning or very poorly functioning neural structures should be resected. By taking nonstimulatable fascicles, one takes either nonfunctioning motor fibers or (possibly functioning) sensory fibers. If the only pathologically appearing fascicles are functioning motor fascicles and the need for a representative sample of the disease is high (as with suspicion of malignancy without the possibility to biopsy elsewhere), then we advocate for taking a motor fascicle from the tibial division rather than the peroneal if both are pathologically involved. The tibial division is larger with more fascicles and is less likely to produce a major motor deficit due to muscle synergism. We also recommend that a small part of the first fascicle be sent for perioperative frozen sectioning. If the first fascicle is confirmed to be diagnostic and an appropriate treatment is initiated, then the remaining nerve can be left alone and potentially might gain some or all of its previous function.

\section{Lessons Learned}

Over our 15-year-long period of accumulating experience, we have learned several key points that warrant further discussion.

Based on our experience correlating MRI appearance with biopsy results, we have identified several entities that present with reproducible imaging and clinical patterns such as inflammatory demyelination (often CIDP), perineurioma, lipomatosis of nerve, neurolymphomatosis, ${ }^{5}$ and neuromuscular choristoma, which can, when classic features are present, be diagnosed without biopsy. Furthermore, in some cases, biopsy itself is not entirely benign and 
may have unintended consequences, such as the desmoid tumors seen after biopsy of neuromuscular choristoma. When evaluating MRI and selecting the biopsy site, not all abnormally appearing nerves on imaging harbor pathology; in some cases, the abnormal appearance of a nerve may reflect a downstream effect from more proximal pathology such as spondylosis in the lumbar spine, so correlation with all available information is critical. If the disease affects more than 1 segment of the nerve, always choose the less risky approach. During the procedure itself harvest only nonstimulating fascicles and resect at their maximal length (the disease can cause "skip lesions"). Pay attention to the appearance of the fascicles: only some fascicles may harbor diagnostic pathology, while the other neighboring fascicles can be completely normal. If the appearance is equivocal, tibial division is preferred over the peroneal. Overall, some diagnoses thought to be rare were actually more prevalent than previously considered (although this is likely caused by the selection bias of a superspecialized tertiary institution). Also, biopsies helped to refine the mechanisms of disease, such as perineural spread of prostate or cervical cancer. ${ }^{10,13,14}$ Even for more prevalent diagnoses, the biopsy allows for more targeted and aggressive treatment.

\section{Future Directions}

The procedure we have used is readily performed and could be adapted more widely. We recognize that, as in any other surgery, the goal is to improve safety and efficacy. The safest surgery is no surgery, and one should avoid unnecessary biopsies. In those instances where tissue is needed, the goal is to select the single best fascicle. "Best" means a fascicle that will not produce a deficit and will provide diagnosis. Safety could be improved by implementing more advanced electrophysiological testing. This might include intraoperative nerve action potential recordings, microelectrode recordings, or needle electromyography. However, the benefits of more invasive monitoring and prolonging a relatively straightforward procedure such as nerve biopsy have yet to be evaluated.

The more precise localization of pathology and selection of an affected fascicle could be enhanced preoperatively by utilizing more advanced imaging such as MR spectroscopy or diffuse-tensor imaging. Ultrasound, either preoperatively or intraoperatively, could provide information of the nerve ultrastructure in greater detail than MRI. Other forms of intraoperative navigation could be represented by a radio, color, or fluorescent tracer that could help to localize and delineate the pathology in a similar manner as 5-aminolevulinic acid is used for brain tumors or radioactive albumin to identify lymph nodes.

\section{Conclusions}

We present a series of 112 targeted fascicular biopsies of the sciatic nerve, which yielded a diagnosis in $84.8 \%$ of cases. The diagnoses were very diverse, and on several occasions the biopsy revealed a diagnosis considered very unlikely in the differential diagnosis. This high diagnostic rate was frequent for treatable conditions and resulted in the patient's appropriate therapy. Significant complications were seen in only 3 patients $(2.7 \%)$ with mild sensory deficits and 2 patients (1.8\%) who developed desmoid tumors after biopsy for neuromuscular choristoma. Because of our experience with a large number of patients with biopsyproven results, we have identified new radiological patterns of disease (including inflammatory demyelination [often CIDP], ${ }^{3}$ perineurioma, ${ }^{3,16}$ lipomatosis of nerve, ${ }^{15}$ and neuromuscular choristoma ${ }^{17}$ ), which has allowed us to forgo biopsy in cases in which classic imaging features are present.

\section{Acknowledgment}

Dr. Capek is supported by European Regional Development Fund-Project FNUSA-ICRC (no. CZ.1.05/1.1.00/02.0123).

\section{References}

1. Abouzahr MK, Lange DJ, Latov N, Olarte M, Rowland LP, Hays AP, et al: Diagnostic biopsy of the motor nerve to the gracilis muscle. Technical note. J Neurosurg 87:122-124, 1997

2. Agadi JB, Raghav G, Mahadevan A, Shankar SK: Usefulness of superficial peroneal nerve/peroneus brevis muscle biopsy in the diagnosis of vasculitic neuropathy. J Clin Neurosci 19:1392-1396, 2012

3. Amrami KK, Felmlee JP, Spinner RJ: MRI of peripheral nerves. Neurosurg Clin N Am 19:559-572, vi, 2008

4. Amrami KK, Spinner RJ, Dyck PJB, Felmlee JP: Techniques for magnetic resonance imaging of peripehral nerves: diagnosis and guidance for targeted fascicular nerve biopsy, in Dyck PJ et al (eds): Companion to Peripheral Neuropathy: Illustrated Cases and New Developments. Philadelphia: Saunders Elsevier, 2010, pp 15-18

5. Capek S, Hebert-Blouin MN, Puffer RC, Martinoli C, Frick MA, Amrami KK, et al: Tumefactive appearance of peripheral nerve involvement in hematologic malignances: a new imaging association. Skeletal Radiol 44:1001-1009, 2015

6. Dy CJ, Lange DJ, Jones KJ, Garg R, DiCarlo EF, Wolfe SW: Diagnostic biopsy of the pronator teres and a motor branch of the median nerve: indications and technique. J Hand Surg Am 37:2570-2575, 2012

7. Dyck PJ, Lofgren EP: Method of fascicular biopsy of human peripheral nerve for electrophysiologic and histologic study. Mayo Clin Proc 41:778-784, 1966

8. Dyck PJB, Spinner RJ, Amrami KK, Klein CJ, Engelstad JK, Dyck PJ: MRI-targeted fascicular nerve biopsies of proximal nerves: historic reports and illustrative case reports, in Dyck PJ, et al (eds): Companion to Peripheral Neuropathy: Illustrated Cases and New Developments. Philadelphia: Saunders Elsevier, 2010, pp 3-14

9. Gabriel CM, Howard R, Kinsella N, Lucas S, McColl I, Saldanha G, et al: Prospective study of the usefulness of sural nerve biopsy. J Neurol Neurosurg Psychiatry 69:442-446, 2000

10. Hébert-Blouin MN, Amrami KK, Myers RP, Hanna AS, Spinner RJ: Adenocarcinoma of the prostate involving the lumbosacral plexus: MRI evidence to support direct perineural spread. Acta Neurochir (Wien) 152:1567-1576, 2010

11. Hébert-Blouin MN, Amrami KK, Spinner RJ: Addendum: Evidence supports a "no-touch" approach to neuromuscular choristoma. J Neurosurg 119:252-254, 2013

12. Hébert-Blouin MN, Scheithauer BW, Amrami KK, Durham SR, Spinner RJ: Fibromatosis: a potential sequela of neuromuscular choristoma. J Neurosurg 116:399-408, 2012

13. Howe BM, Amrami KK, Nathan MA, Garcia JJ, Spinner RJ: Perineural spread of cervical cancer to the sciatic nerve. Skeletal Radiol 42:1627-1631, 2013

14. Ladha SS, Spinner RJ, Suarez GA, Amrami KK, Dyck PJ: Neoplastic lumbosacral radiculoplexopathy in prostate cancer by direct perineural spread: an unusual entity. Muscle Nerve 34:659-665, 2006 
15. Marom EM, Helms CA: Fibrolipomatous hamartoma: pathognomonic on MR imaging. Skeletal Radiol 28:260 264, 1999

16. Mauermann ML, Amrami KK, Kuntz NL, Spinner RJ, Dyck PJ, Bosch EP, et al: Longitudinal study of intraneural perineurioma-a benign, focal hypertrophic neuropathy of youth. Brain 132:2265-2276, 2009

17. Niederhauser BD, Spinner RJ, Jentoft ME, Everist BM, Matsumoto JM, Amrami KK: Neuromuscular choristoma: characteristic magnetic resonance imaging findings and association with post-biopsy fibromatosis. Skeletal Radiol 42:567-577, 2013

18. Oberlin C, Durand S, Belheyar Z, Shafi M, David E, Asfazadourian $\mathrm{H}$ : Nerve transfers in brachial plexus palsies. Chir Main 28:1-9, 2009

19. Oh SJ: Diagnostic usefulness and limitations of the sural nerve biopsy. Yonsei Med J 31:1-26, 1990

20. Rajshekhar V: Current status of stereotactic biopsy. Stereotact Funct Neurosurg 76:137-139, 2001

21. Ruth A, Schulmeyer FJ, Roesch M, Woertgen C, Brawanski A: Diagnostic and therapeutic value due to suspected diagnosis, long-term complications, and indication for sural nerve biopsy. Clin Neurol Neurosurg 107:214-217, 2005

22. Spinner RJ, Amrami KK, Dyck PJB: Targeted fascicular biopsy: a surgical perspective, in Dyck PJ, et al (eds): Companion to Peripheral Neuropathy: Illustrated Cases and New Developments. Philadelphia: Saunders Elsevier, 2010, pp 19-24

\section{Disclosure}

The authors report no conflict of interest concerning the materials or methods used in this study or the findings specified in this paper.

\section{Author Contributions}

Conception and design: Spinner, Capek, Amrami. Acquisition of data: all authors. Analysis and interpretation of data: all authors. Drafting the article: all authors. Critically revising the article: all authors. Reviewed submitted version of manuscript: all authors. Approved the final version of the manuscript on behalf of all authors: Spinner. Statistical analysis: Capek. Administrative/technical/material support: Spinner. Study supervision: Spinner.

\section{Supplemental Information Videos}

Video 1, Media Player. http://mfile.akamai.com/21490/wmv/ digitalwbc.download.akamai.com/21492/wm.digitalsource-naregional/focus15-213_video_1.asx.

Video 1, Quicktime. http://mfile.akamai.com/21488/mov/ digitalwbc.download.akamai.com/21492/qt.digitalsource-global/ focus15-213_video_1.mov.

\section{Correspondence}

Robert J. Spinner, Mayo Clinic, 200 First St. SW, Gonda 8-214, Rochester, MN 55905. email: spinner.robert@mayo.edu. 